\title{
Early Allograft Dysfunction to Assess the Liver Function following Liver Transplantation
}

\author{
Ifeoluwa Bolujo, $\mathrm{MS1}^{1}$, Mohammad Aref, MS4${ }^{1}$, Chandrashekhar A. Kubal, MD, PhD², \\ Richard S. Mangus, $\mathrm{MD}^{2}$, Burcin Ekser, $\mathrm{MD}, \mathrm{PhD}^{2}$ \\ (1) Indiana University School of Medicine; (2) Transplant Surgery, Department of Surgery, \\ Indiana University School of Medicine
}

Background: Early allograft disfunction (EAD) can be used as a tool to asses function of the liver after transplantation. Currently, most accepted EAD definition can be defined using a number of criteria including but not limited to total bilirubin $>10 \mathrm{mg} / \mathrm{dL}$ on post-operative day 7 , INR > 1.6 on post-operative day 7, and ALT or AST > 2000 IU/L within the first post-operative 7 days. EAD is associated with mortality and morbidity after liver transplantation which leads to decreased recipient survival rates. It is considered a precursor step in the pathway to eventual graft loss.

Methods: We have performed a literature review to understand what risk factors increase, including donor and recipient demographics as well as recipients' MELD (Model for End Stage Liver Disease) scores, which predicts patients' disease severity. We also compared the incidence of EAD between our center and the literature.

Results: We found that incidence of EAD was between $23-40 \%$ (Table 2). It is higher when livers from donation after circulatory death (DCD) donors were used, as expected.

\begin{tabular}{|l|l|l|}
\hline Reference (year) & Number of patients & Incidence of EAD \\
\hline Ekser et al (2019) & $\mathrm{n}=2008$ & $29 \%$ \\
\hline Olthoff et al (2010) & $\mathrm{n}=300$ & $23 \%$ \\
\hline Wadei et al (2015) & $\mathrm{n}=1325$ & $27 \%$ \\
\hline Lee et al (2014) & $\mathrm{n}=205$ & $39.5 \%$ (DCD donors) \\
\hline
\end{tabular}

Risk factors that can result in an increase occurrence of EAD were; (i) donor age, (ii) liver stenosis, (iii) expanding criteria for donor livers, (iv) prolonged warm ischemia time (WIT) and (v) cold ischemia time (CIT). Histologically, hepatocellular damage that is shown as coagulative necrosis can be seen immediately after reperfusion.

Conclusions: During our literature review, potential strategies have been discovered to help prevent EAD including pharmacological treatment using glucose, antioxidants, antiinflammatories, and apoptotic drugs. Although none of them significantly prevented the occurrence of EAD, it was clear that there are many tools that can be used to attempt to prevent $E A D$ in order to offer a better outcome in patients undergoing liver transplantation. 